\title{
SUBOBJECT CLASSIFIERS AND CLASSES OF SUBFUNCTORS1)
}

\author{
HANS ENGENES2)
}

The purpose of this paper is to show that for certain categories $\mathscr{S}$ of sets, including the category of all sets for those readers who do not let set-theoretic difficulties stop them, the functor categories

$$
\mathscr{S}^{\mathscr{S}} \text { and } \mathscr{S}^{\mathscr{S} \text { op }}
$$

both have subobject classifiers (Corollary 1), but are not cartesian closed (Corollary 2).

We say a category $\mathscr{C}$ is a full category of sets if the objects of $\mathscr{C}$ are sets, each of its hom-sets of the form $\mathscr{C}(A, B)$ is the set of all functions from $A$ to $B$, and composition rules and identity maps are as usual for functions between sets. We denote by $\mathscr{S}$ any full category of sets whose object class $|\mathscr{S}|$ has the following property:

(*) If $|\mathscr{S}|$ has members of cardinalities $\alpha$ and $\beta$, and if $\gamma<\alpha$, then $|\mathscr{S}|$ has members of cardinalities $\gamma, \alpha \cdot \beta$, and $2^{\alpha}$.

Some examples of what $|\mathscr{S}|$ can possibly be are: all sets, all sets of cardinality or rank less than some given strong limit cardinal $x$ (meaning: $\gamma<x \Rightarrow 2^{\gamma}<x$ ), all cardinal numbers smaller than such a $x$, any universe in the sense of Grothendieck-Bourbaki (see [2]), but not any school of sets in the sense of Mac Lane [5]. We assume the axiom of choice (hereafter called $\mathrm{AC}$ ) in the form that onto functions between sets have sections, or right inverses. In any full category of sets epimorphisms are onto, so any such category satisfies the categorical form of $\mathrm{AC}$, in the sense of [10].

We will say that a full category of sets is rich if it is closed under formation of subsets, power sets, finite cartesian products (constructed in the usual set theoretic manner), and sets of all functions between any two

Received August 7, 1973.

1) This paper is the result of inspiring conversations and discussions with George $D$. Reynolds, the author's colleague, and Fred E. J. Linton, the author's thesis advisor.

2) The author was supported by a graduate student stipend from Wesleyan University while writing this paper.

Math. Scand. $84-10$ 
members. In an attempt to avoid foundational difficulties of a set theoretic nature, we will not assume that a category of all sets is available as a mathematical object, but we will assume that any full category of sets can be extended to a rich category of sets. It is easy to see that $\mathscr{S}$, satisfying.:(*), has a rich extension which is equivalent to $\mathscr{S}_{\text {: as a cate- }}$ gory. So we can assume that $\mathscr{S}$ is rich, since the statements to be proved in this paper are about properties which are preserved under equivalences of categories. Furthermore we will assume that for any given category $\mathscr{X}$ there is a full category of sets containing the hom-sets of $\mathscr{X}$, and one containing, for each $X \in|\mathscr{X}|$, the class of all subobjects of $X$ (see definition below).

We will need the following definitions: A set (or class) is $\mathscr{S}$-small if its cardinality is equal to that of an $\mathscr{S}$-object. A category is locally $\mathscr{S}$-small if all its hom-sets are $\mathscr{S}$-small, and a locally $\mathscr{S}$-small category is $\mathscr{S}$-small if its object class is $\mathscr{S}$-small. A subobject of an object $X$ in a category is an equivalence class of monomorphisms into $X$. If $\mathscr{X}$ is a category with pullbacks, the power object functor on $\mathscr{X}$ is the contravariant functor which associates to any object its class of subobjects, and to any morphism $f: X \rightarrow Y$ the operation of pulling back subobjects of $Y$ along $f$. If this functor is representable, then any representing object for it is called a subobject classifier in $\mathscr{X}$. A category $\mathscr{X}$ with finite products is cartesian closed if for any object $X$ the functor $-\times X: \mathscr{X} \rightarrow \mathscr{X}$ has a right adjoint. Finally, $\mathscr{X}$ is a topos if it has all finite limits, is cartesian closed, and has a subobject classifier (hence has all finite colimits, see [7] or [8]).

As Freyd has pointed out in [1], the universal monomorphism $\Omega^{\prime} \succ \Omega$ into a subobject classifier $\Omega$, mediating the representation of the power object functor, must have $\Omega^{\prime}$ terminal in the category. This provides the connection with the more familiar (and equivalent) definition: $\Omega$ is a subobject classifier by way of a map $t$ from the terminal object $T$ to $\Omega$, if for any monomorphism $f: X \succ Y$ there is a unique map $\varphi: Y \rightarrow \Omega$ making $!_{X}: X \rightarrow T$ and $f$ the pullback of $t$ and $\varphi$.

It is easy to see that $\mathscr{S}$ is a topos, and it is well-known that $\mathscr{S}^{\mathscr{X}}$ also is when $\mathscr{X}$ is $\mathscr{S}$-small. If $\mathscr{S}^{\mathscr{X}}$ is to have a subobject classifier $\Omega$, then, for any $X \in|\mathscr{X}|$, the class of subfunctors of $\mathscr{X}(X,-)$ must be $\mathscr{S}$-small, being in bijective correspondence with $\mathscr{S}^{\mathscr{X}}(\mathscr{X}(X,-), \Omega)$ (assume for a moment that the hom-sets of $\mathscr{X}$ are in $\mathscr{S}$ ) and hence with $\Omega(X)$. And if

$$
-\times F: \mathscr{S}^{\mathscr{X}} \rightarrow \mathscr{S}^{\mathscr{X}}
$$

has a right adjoint $[F,-]$, then, for any $X \in|\mathscr{X}|$, the class of natural 
transformations from $\mathscr{X}(X,-) \times F$ to any $G \in\left|\mathscr{S}^{\mathscr{X}}\right|$ must be $\mathscr{S}$-small, being in bijective correspondence with

$$
\mathscr{S}^{\mathscr{X}}(\mathscr{X}(X,-),[F, G])
$$

and hence with $[F, G](X)$. Inspection of the proofs in [1] or [9] that $\mathscr{S}^{\mathscr{X}}$ is a topos for $\mathscr{S}$-small $\mathscr{X}$ reveals that these two necessary conditions, obviously satisfied when $\mathscr{X}$ is $\mathscr{S}$-small, are in fact sufficient; in effect, what is really proved is:

Lemma 1. Let $\mathscr{S}$ be as described, let $\mathscr{X}$ be a locally $\mathscr{S}$-small category, and let $\mathscr{S}^{\prime}$ be a rich extension of $\mathscr{S}$ containing all the hom-sets of $\mathscr{X}$ but no sets larger than those in $|\mathscr{S}|$. Then $\mathscr{S}^{\mathscr{X}}$ has a subobject classifier if and only if the class of all subfunctors of each representable functor $\mathscr{X} \rightarrow \mathscr{S}^{\prime}$ is $\mathscr{S}$-small, and $\mathscr{S}^{\mathscr{X}}$ is cartesian closed if and only if the class

$$
\mathscr{S}^{\prime} \mathscr{X}(\mathscr{X}(X,-) \times F, G)
$$

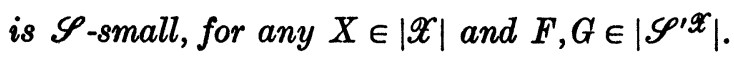

We remark here, for future reference, that for a locally $\mathscr{S}_{\text {-small cate- }}$ gory $\mathscr{X}$ with an initial object $I$, the category $\mathscr{S}^{\mathscr{X}}$ is cartesian closed if

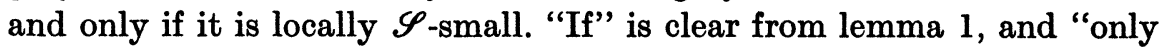
if" follows from lemma 1 and the observation that each set-valued functor $F$ on $\mathscr{X}$ is naturally equivalent to $\mathscr{X}(I,-) \times F$. If $\mathscr{X}$ does not have an initial object, virtually anything can happen. E.g. putting $\mathscr{X}=|\mathscr{S}|$ - the discrete category on the objects of $\mathscr{S}$ - yields an example where $\mathscr{S}^{\mathscr{X}}$ is a topos but not locally $\mathscr{S}$-small: Each representable functor has exactly two subfunctors, and the natural transformations from

$$
|\mathscr{S}|(X,-) \times F
$$

to $G$ are in bijective correspondence with the $\mathscr{S}$-small set of all functions from $F(X)$ to $G(X)$. So $\mathscr{S}^{|\mathscr{S}|}$ is a topos by lemma 1 , and it is not locally $\mathscr{S}$-small because the inclusion functor $|\mathscr{S}| \rightarrow \mathscr{S}$ has a non- $\mathscr{S}$-small class of natural transformations to itself (this follows from the obvious fact that $\mathscr{S}$ has no object of maximal cardinality). Now $\mathscr{S}^{|\mathscr{S}|}$ is just a product of toposes, each of the form $\mathscr{S}$. It is not hard to show that given any family of toposes, its product category is again a topos. So if $\mathscr{X}$ is a locally $\mathscr{S}$-small groupoid (all morphisms invertible) then $\mathscr{S}^{\mathscr{X}}$ is a topos, being equivalent to a product of categories of the form $\mathscr{S}^{M}$, $M$ an $\mathscr{S}$-small group. Next let $\mathscr{X}$ be the (non- $\mathscr{S}$-small) partially ordered category of all $\mathscr{S}$-small ordinals. Then any representable functor $\mathscr{X} \rightarrow \mathscr{S}$ has a non- $\mathscr{S}$-small class of subfunctors - the representable subfunctors 
alone are too numerous (by the way, each subfunctor is representable) so $\mathscr{S}^{\mathscr{X}}$ has no subobject classifier. Nor is $\mathscr{S}^{\mathscr{X}}$ locally $\mathscr{S}$-small, and hence not cartesian closed, for (assuming for simplicity that each $\mathscr{S}$-small ordinal is an $\mathscr{S}$-object) the natural transformations from the inclusion functor $\mathscr{X} \rightarrow \mathscr{S}$ to itself are in bijective correspondence with the nonincreasing functions from the well-ordered class $|\mathscr{X}|$ into itself, and they form a non- $\mathscr{S}$-small class. In contrast to all this, $\mathscr{S}^{\mathscr{X} \text { op }}$ is a topos, as the reader can easily verify.

In the following we will show that, when $\mathscr{X}$ is $\mathscr{S}$ or $\mathscr{S}^{\text {op }}, \mathscr{S}^{\mathscr{X}}$ has a subobject classifier, but is not locally $\mathscr{S}$-small, hence not cartesian closed, hence not a topos.

We will use the assumption, as mentioned before, that $\mathscr{S}$ is actually a rich category of sets. Then subobjects in $\mathscr{S}$ have canonical representatives (inclusions of subsets), and the same is true for $\mathscr{S}^{\mathscr{X}}$ (inclusions of subfunctors). Notice that to define a subfunctor $G$ of an $\mathscr{S}$-valued functor $F$ on $\mathscr{X}$, we need only define subsets $G(X) \subset F(X)$, for each $X \in|\mathscr{X}|$, in such a manner that for any $\mathscr{X}$-morphism $f: X \rightarrow Y, F(f)$ maps the set $G(X)$ into $G(Y)$. We write $G \subset F$ to express that $G$ is a subfunctor of $F$.

Proposition 1. For any set $X \in|\mathscr{S}|$ the subfunctors of $\mathscr{S}(X,-)$ : $\mathscr{S} \rightarrow \mathscr{S}$ form an $\mathscr{S}$-small set, and so do the subfunctors of $\mathscr{S} \circ \mathrm{p}(X,-)=$ $\mathscr{S}(-, X): \mathscr{S}_{\mathrm{op}}^{\rightarrow} \mathscr{S}$.

Proof. We deal first with the covariant case. If $X=\varnothing$ then $\mathscr{S}(X,-)$ has exactly three subfunctors: the empty functor, the functor $F$ defined by $F(\varnothing)=\varnothing$ and $F(Y)=\mathscr{S}(\varnothing, Y)=\left\{!_{Y}\right\}$ for $Y \neq \varnothing$, and then $\mathscr{S}(\varnothing,-)$ itself.

So now assume that $X \neq \varnothing$. It suffices to show that a given subfunctor $F \subset \mathscr{S}(X,-)$ is determined by its action on $X$, i.e. by the subset $F(X) \subset$ $\mathscr{S}(X, X)$. Since $\mathscr{S}(X, \varnothing)$ is empty, so is $F(\varnothing)$. If $Y \in|\mathscr{S}|$ is nonempty, and $|Y| \leq|X|$, then let $j: Y \hookrightarrow X$ be a monomorphism. Since there is $p: X \rightarrow Y$ satisfying $p \circ j=\mathrm{id}_{Y}$, it is easy to see that

$$
F(Y)=\{f \in \mathscr{S}(X, Y) \mid j \circ f \in F(X)\} .
$$

If $|Y|>|X|$, let $i: X \hookrightarrow Y$ be a one-to-one map, and let $r: Y \rightarrow X$ be such that $r \circ i=\mathrm{id}_{X}$. For any $g \in \mathscr{S}(X, Y)$ we can obtain a bijection $h_{g}: Y \rightarrow Y$ with $h_{g}(g(X)) \subset i(X)$ as follows. On $g(X)$ let $h_{g}$ be any one-toone map of $g(X)$ into $i(X)$. Then note that $Y \backslash g(X)$ and $Y \backslash h_{g}(g(X))$ have the same cardinality, so take any bijection of the former onto the latter as the action of $h_{g}$ on $Y \backslash g(X)$. Notice that $g \in F(Y)$ if and only if $h_{g} \circ g \in F(Y)$, since $h_{g}$ is invertible. And since $h_{g} \circ g$ maps $X$ into $i(X)$, 
there is (by AC) a function $f_{g}: X \rightarrow X$ such that $h_{g} \circ g=i \circ f_{g}$. Now we can see that

$$
F(Y)=\left\{g \in \mathscr{S}(X, Y) \mid r \circ h_{g} \circ g \in F(X)\right\} \text {. }
$$

The inclusion " $\subset$ " is clear, and for the converse suppose $r \circ h_{\sigma} \circ g \in F(X)$. Then

$$
h_{g} \circ g=i \circ f_{g}=i \circ r \circ i \circ f_{g}=i \circ r \circ h_{g} \circ g \in F(Y),
$$

so $g \in F(Y)$ as desired. So $F(Y)$ is determined by $F(X)$, for any $Y \in|\mathscr{S}|$, and this completes the proof of the covariant case.

Before we prove the contravariant case we need one more definition and some more notation. A quasifilter on a set $X$ will be a (possibly empty) family of subsets of $X$ (all of them nonempty, if $X$ is nonempty), containing all nonempty subsets of each of its members. We write $Q(X)$ for the set of all quasifilters on $X$. The empty functor $\mathscr{S}^{\text {op }} \rightarrow \mathscr{S}$ is denoted by $F_{0}$, it is a subfunctor of any $\mathscr{S}$-valued functor on $\mathscr{S}$ op. We write $F_{1}$ for the subfunctor of $\mathscr{S}(-, X)$ defined by

$$
F_{1}(Y)=\varnothing \Leftrightarrow Y \neq \varnothing
$$

(so $F_{1}$ depends on the set $X$ ). Finally, we write $\Psi(X)$ for the class of subfunctors of $\mathscr{S}(-, X): \mathscr{S}_{\mathrm{op}} \rightarrow \mathscr{S}$. If $X$ is an $\mathscr{S}$-small set then clearly so is $Q(X)$. We will show that $\Psi(X)$ also is $\mathscr{S}$-small, by putting it in "almost" bijective correspondence with $Q(X)$. We note in passing that $F_{0} \neq F_{1}$ for any set $X$, and if $F \in \Psi(X) \backslash\left\{F_{0}\right\}$ then $F_{1} \subset F$. So $\Psi(X)$ has a smallest and a second smallest element in the partial ordering " $\subset$ ".

One can easily verify that the following formulas define mappings $a: \Psi(X) \rightarrow Q(X)$ and $b: Q(X) \rightarrow \Psi(X):$

$$
\begin{aligned}
a(F) & =\{f(X) \mid f \in F(X)\} \quad \text { for } F \in \Psi(X), \\
b(J)(Y) & =\{f \in \mathscr{S}(Y, X) \mid \exists U \in J: f(Y) \subset U\} \quad \text { for } J \in Q(X), Y \in|\mathscr{S}| .
\end{aligned}
$$

Now, to wrap up the proof of proposition 1, we need only prove:

Lemma 2. For any set $X, a \circ b=\mathrm{id}_{Q(X)}$. If $F \in \Psi(X)$ and either $F \neq F_{1}$ or $X=\varnothing$ then $b(a(F))=F$. If $X \neq \varnothing$ then $b\left(a\left(F_{1}\right)\right)=F_{0}$.

REMaRK. Thus $Q(X)$ is in bijective correspondence with $\Psi(X) \backslash\left\{F_{1}\right\}$, if $X \neq \varnothing$, or with $\Psi(X)$, if $X=\varnothing$, so $\Psi(X)$ is an $\mathscr{S}$-small set whenever $X$ is.

Proof of Lemma 2. If $X=\varnothing$ then

$$
\begin{array}{rlrl}
\Psi(X)=\left\{F_{0}, F_{1}\right\}, & Q(X) & =\{\varnothing,\{\varnothing\}\}, \\
a\left(F_{0}\right)=\varnothing, & a\left(F_{1}\right)=\{\varnothing\}, & b(\varnothing) & =F_{0} \quad b(\{\varnothing\})=F_{1},
\end{array}
$$

so everything is as it should be. 
Now assume $X \neq \varnothing$, and let $J \in Q(X)$. To see that $a(b(J))=J$ we show inclusion both ways. If $f(X) \in a(b(J))$ with $f \in b(J)(X)$, then $f(X)$ is a nonempty subset of a member of $J$, so $f(X) \in J$. Conversely, if $U \in J$ then $\varnothing \neq U \subset X$, so there is a function $f: X \rightarrow X$ mapping $X$ onto $U$, and any such $f$ is in $b(J)(X)$, so $U=f(X) \in a(b(J))$. Hence $a(b(J))=J$, as desired.

Next let $F \in \Psi(X) \backslash\left\{F_{1}\right\}$, and let $Y \in|\mathscr{S}|$. To see that $b(a(F))(Y)=$ $F(Y)$, we show inclusion both ways. If $f \in b(a(F))(Y)$ then there is $g \in F(X)$ with $f(Y) \subset g(X)$, by definitions of $a$ and $b$. Then, by AC, $f$ factors through $g$, i.e. there is $h: Y \rightarrow X$ with $g \circ h=f$. So $f \in F(Y)$. Conversely, if $f \in F(Y)$, then $F(Y) \neq \varnothing$ so $F \neq F_{0}$. Since also $F \neq F_{1}$, it follows that $a(F) \neq \varnothing\left(F_{0}\right.$ and $F_{1}$ are the only subfunctors of $\mathscr{S}(-, X)$ taking the value $\varnothing$ at $X$ ), so $b(a(F))(Y) \neq \varnothing$. If $Y=\varnothing$, then $b(a(F))(Y)$, being a nonempty subset of $\mathscr{S}(\varnothing, X)=\left\{!_{X}\right\}$, certainly contains $f$. And if $Y \neq \varnothing$ there will be some $h: X \rightarrow Y$ with $f(h(X))=f(Y)$ (by AC), and $f \circ h \in$ $F(X)$, so $f(Y) \in a\left(F^{\prime}\right)$, so $f \in b(a(F))(Y)$. Hence $b(a(F))=F$, as desired.

Finally, since $X \neq \varnothing$ we have $a\left(F_{1}\right)=\varnothing$, so $b\left(a\left(F_{1}\right)\right)=b(\varnothing)=F_{0}$. This completes the proof of lemma 2 , and hence of proposition 1.

Corollary 1. $\mathscr{S}^{\mathscr{S}}$ and $\mathscr{S}^{\mathscr{S} \text { op }}$ both have subobject classifiers.

Proor. Having assumed, without loss of generality, that $\mathscr{S}$ is rich, lemma 1 and proposition 1 apply directly.

We will denote the subobject classifiers in $\mathscr{S}^{\mathscr{S}}$ and $\mathscr{S}^{\mathscr{S} \text { op }}$ by $\Omega$ and $\Psi$ respectively. This is consistent with the use we have made of the symbol $\Psi$ in the proof of proposition 1 above. Let $P$ denote the covariant power set functor $\mathscr{S} \rightarrow \mathscr{S}$, which takes a set to its power set and a function $f: X \rightarrow Y$ to the "direct image under $f$ " operation $P(X) \rightarrow P(Y)$ (assuming, as before, that $\mathscr{S}$ is rich). Let $K$ denote the functor

$$
\mathscr{S}(P(-), 2): \mathscr{S}_{\mathrm{op}}^{\rightarrow} \mathscr{S},
$$

where $2=\{0,1\}$ is some two-element set in $|\mathscr{S}|$. Essentially, $K$ is the composition of the covariant with the contravariant power set functor. By definition, a category is $\mathscr{S}$-well-powered if each of its objects has only an $\mathscr{S}$-small class of subobjects.

Proposition 2. The subfunctors of $P$, as well as those of $K$, form non$\mathscr{S}$-small classes, so neither $\mathscr{S}^{\mathscr{S}}$ nor $\mathscr{S}^{\mathscr{S} \text { op }}$ is $\mathscr{S}$-well-powered.

Proor. For $P$ it suffices to observe that for any cardinal number $\alpha$,

$$
P_{\alpha}(X)=\{Y \subset X|| Y \mid<\alpha\}
$$


defines a subfunctor $P_{\alpha}$ of $P$, distinct $\mathscr{S}_{\text {-small cardinals define dìstinct }}$ subfunctors, and the $\mathscr{S}$-small cardinal numbers do not form an $\mathscr{S}$-small set.

For $K$, we map the class of subfunctors of $P$ in a one-to-one fashion into the class of subfunctors of $K$. Given $H \subset P$, let us define $A \subset K$ by

$$
\hat{H}(X)=\{f \in \mathscr{S}(P(X), 2)|f| H(X) \equiv 1\} .
$$

To see that this indeed defines a subfunctor $\hat{H}$ of $K$, let $g \in \mathscr{S}(X, Y)$. If $f=h \circ P(g) \in K(g)(\hat{H}(Y))$ with $h \mid H(Y) \equiv 1$, then

$$
f|H(X)=(h \circ P(g))| H(X) \equiv 1
$$

because $P(g)$ maps $H(X)$ into $H(Y)$. Hence $K(g)(\hat{H}(Y)) \subset \widehat{H}(X)$, as desired.

Finally, if $H_{1} \neq H_{2} \subset P$, say if $H_{1} \notin H_{2}$, then there is $X \in|\mathscr{S}|$ with $H_{1}(X) \notin H_{2}(X)$. Then the function $f: P(X) \rightarrow 2$ which is constantly 1 on $H_{2}(X)$ and constantly 0 on $P(X) \backslash H_{2}(X)$ is in $\hat{H}_{2}(X)$ but not in $\hat{H}_{1}(X)$. So $\hat{H}_{1} \neq \hat{H}_{2}$, as desired.

Conollary 2. Neither $\mathscr{S}^{\mathscr{S}}$ nor $\mathscr{S}^{\mathscr{S} \text { op }}$ is locally $\mathscr{S}_{\text {-small, nor is either }}$ cartesian closed, nor, hence, is either a topos.

Proof. By corollary 1, each of these categories has a subobject classifier, but by proposition 2 , neither is $\mathscr{S}$-well-powered, so neither can be locally $\mathscr{S}$-small. Since $\mathscr{S}$ and $\mathscr{S}$ op both have initial objects, the rest is dealt with by the remark immediately after lemma 1.

\section{BIBLIOGRAPHY}

1. P. Freyd, Aspects of topoi, Bull. Austral. Math. Soc. 7 (1972), 1-76.

2. A. Grothendieck and J. L. Verdier, Prefaisceaux (with an appendix by N. Bourbaki), in Théorie des Topos et Cohomologie Etale des Schémas (SGA 4), Lecture Notes in Mathematics 269, 1-217, Springer-Verlag, Berlin, Heidelberg, New York, 1972.

3. A. Kock and G. C. Wraith, Elementary Toposes, Aarhus University Lecture Notes Series in Mathematics 30, Aarhus, 1971.

4. F. W. Lawvere, Introduction to Toposes, Algebraic Geometry and Logic, Lecture Notes in Mathematics 274, 1-12, Springer-Verlag, Berlin, Heidelberg, New York, 1972.

5. S. MacLane, Foundations for categories and sets, in Category Theory, Homology Theory and their Applications II, ed. by P. Hilton, Lecture Notes in Mathematics 92, 146164, Springer-Verlag, Berlin, Heidelberg, New York, 1969.

6. S. MacLane, Categories for the Working Mathematician, Graduate Texts in Mathematics 5, Springer-Verlag, New York, Heidelberg, Berlin, 1971.

7. C. J. Mikkelsen, Finite colimits in toposes, Talk at the conference on category theory at Oberwolfach, July 1972.

8. R. Paré, Colimits in topoi, preprint, Dalhousie University, Halifax, Nova Scotia, 1973. 
9. M. Tierney, Axiomatic sheaf theory: some conctructions and applications, Lectures delivered at C.I.M.E. Conference on Category Theory and Commutative Algebra, Varenna, Italy, 1971.

10. M. Tierney, Sheaf theory and the continuum hypothesis, in Toposes, Algebraic Geometry and Logic, ed. by F. W. Lawvere, Lecture Notes in Mathematics 274, 13-42, Springer-Verlag, Berlin, Heidelberg, New York, 1972.

WESLEYAN UNIVERSITY

MIDDLETOWN, CONNECTICUT, U.S.A. 